\title{
An in vivo model for thyroid regeneration and folliculogenesis
}

\author{
Manabu Iwadate $^{1,2} \cdot$ Yoshinori Takizawa $^{1,3} \cdot$ Yo-Taro Shirai $^{1} \cdot$ Shioko Kimura $\mathbb{D}^{1}$
}

Received: 15 February 2018 / Revised: 23 March 2018 / Accepted: 29 March 2018 / Published online: 26 June 2018

(c) United States \& Canadian Academy of Pathology 2018

\begin{abstract}
While thyroid is considered to be a dormant organ, when required, it can regenerate through increased cell proliferation. However, the mechanism for regeneration remains unknown. Nkx2-1(flfl);TPO-cre mouse thyroids exhibit a very disorganized appearance because their thyroids continuously degenerate and regenerate. In mouse thyroids, a cluster of cells are found near the tracheal cartilage and muscle, which are positive for expression of NKX2-1, the master transcription factor governing thyroid development and function. In the present study, we propose that this cluster of NKX2-1-positive cells may be the precursor cells that mature to become thyroid follicular cells, forming thyroid follicles. We also found that phosphorylation of AKT is induced by NKX2-1 in the proposed thyroid progenitor-like side-population cell-derived thyroid cell line (SPTL) cells, suggesting the possibility that NKX2-1 plays a role in differentiation through the modulation of AKT signaling. This study revealed that $N k x 2-1(f / f l)$;TPO-cre mice provide a suitable model to study in vivo regeneration and folliculogenesis of the thyroid.
\end{abstract}

\section{Introduction}

Thyroid gland is considered to be a dormant organ, and it is estimated that the turnover time of human thyroid gland is approximately 10 years [1]. However, perturbation of the pituitary-thyroid axis as a result of xenobiotic stimuli or physiological alternations can result in cell hypertrophy and proliferation $[2,3]$. The thyroid also responds to hypothyroidism caused by autoimmune diseases or surgical partial thyroidectomy to restore thyroid hormone levels through hyperplasia $[4,5]$. This feedback function of the

These authors contributed equally to this study: Manabu Iwadate, Yoshinori Takizawa.

Electronic supplementary material The online version of this article (https://doi.org/10.1038/s41374-018-0068-x) contains supplementary material, which is available to authorized users.

Shioko Kimura

kimuras@mail.nih.gov

1 Laboratory of Metabolism, National Cancer Institute, National Institutes of Health, Bethesda, MD 20892, USA

2 Department of Thyroid and Endocrinology, Fukushima Medical University, Fukushima 960-1295, Japan

3 Department of Otorhinolaryngology, Seirei Mikatahara General Hospital, Hamamatsu, Shizuoka 433-8558, Japan thyroid was exploited by using partial thyroidectomy in mice as a tool to study thyroid regeneration [6-9].

The $N k x 2-1(f / f l)$;TPO-cre mouse expresses Cre recombinase under control of the thyroid peroxidase promoter, which disrupts the Nkx2-1 gene, a thyroid differentiation marker gene, in a thyroid follicular cells-specific fashion [10]. However, in their thyroids, the $N k \times 2-1$ gene is disrupted in a hypomorphic manner resulting in thyroid follicles that consist of follicular cells having the $N k x 2-1$ gene almost completely disrupted, while other follicular cells have no alterations in the $N k \times 2-1$ gene. As a whole, these thyroids appear to have approximately one-half of the $N k \times 2-1$ alleles deleted [11]. The thyroids of these mice are very disorganized and the follicular cells that have lost NKX2-1 expression appear to undergo degeneration. As a result of this, their thyroids have approximately a 2-fold higher proliferation rate, which contributes to a higher incidence of thyroid tumors induced by genotoxic carcinogen, such as $\mathrm{N}$ bis(2-hydroxypropyl)-nitrosamine (DHPN) [12].

While the presence of stem cells in the thyroid has been documented in mice and humans [13-16], whether stem cells are involved in the process of turnover, hypertrophy, and/or hyperplasia of the thyroid remains largely unknown. To examine the nature of thyroid stem/progenitor cells, we isolated side-population (SP) cells from mouse thyroids [13] and established a cell line called side-population cellderived thyroid cell line (SPTL) [17]. Electron microscopy studies showed that SPTL cells are apparently immature 
cells. Under low-serum differentiation conditions in culture, only a small fraction of SPTL cells expressed NKX2-1 $(\sim 1.3 \%)$, but no other thyroid differentiation marker genes, including Pax8, Foxel, $\mathrm{Tg}$ (thyroglobulin), Tshr (TSH receptor), Tpo (thyroid peroxidase), and Scl5a5 (Nis, sodium iodide symporter). In three-dimensional Matrigel cultures, SPTL cells formed follicle-like structure where none of thyroid differentiation marker genes were expressed. In contrast, SPTL cells that express NKX2-1 were found in part of the follicles after injection to mice [17]. NKX2-1 is known to act as a lineage specification signal $[18,19]$, while both NKX2-1 and PAX8 are required for thyroid follicular cells differentiation [20-22]. Based on these results, it was suggested that SPTL cells may be characterized as partially thyroid-specified progenitor-like cells, which may provide a good tool to study thyroid stem cells [17].

In this study, we demonstrate that a cluster of cells expressing NKX2-1 are present in the Nkx2-l(f//fl);TPO-cre mouse thyroid, which may be precursor cells that can mature to become thyroid follicular cells. This process might involve phosphorylation of AKT induced by NKX21. To our knowledge, this is the first report describing a possible mechanism for thyroid folliculogenesis in mice in vivo. The $N k x 2-1(f l f l)$;TPO-cre mouse provides a possible tool to study regeneration and folliculogenesis.

\section{Materials and methods}

\section{Animals}

The mice used in this study are designated $N k x 2-1(f / f l)$; TPO-cre mice and $N k x 2-1(f / w t)$;TPO-cre littermates as a control on the C57BL/6N background (minimum 6 times backcrossed). The details of these mice were previously described [11]. At the age of 3-4 months (males/females), their thyroids were resected and subjected to histological, immunohistochemical and immunofluorescence analyses. Some mice were intraperitoneally injected with bromodeoxy uridine (BrdU) $(50 \mu \mathrm{g} / \mathrm{g}$ body weight) twice a day for 7 consecutive days, and one day after the last injection, their thyroids were removed for immunohistochemical analysis. EGFP reporter mice (C57BL/6-Tg(CAG-EGFP) $10 \mathrm{sb} / \mathrm{J}$, no. 003291) were obtained from the Jackson Laboratory. All mice were housed in a temperature and humidity controlled specific pathogen-free AAALACaccredited facility under a 12-h light/dark cycle with free access to water and food, and were handled in a humane manner in accordance with the established NIH Guidelines. Animal studies were carried out under protocols approved by the National Cancer Institute Animal Care and Use Committee.

\section{Bone marrow transplant}

Nkx2-1(fl/fl);TPO-cre mice (4-8-week-old) were irradiated (900 cGy) 4-6 h before bone marrow cells transplantation. Bone marrow cells $\left(5 \times 10^{5}\right)$ from EGFP reporter mice (donor mice) were injected into the tail vein of recipient $N k x 2-1(f l f l)$ ); TPO-cre mice (both are on the C57BL/6 background) in $100 \mu \mathrm{l}$ PBS. Autoclaved food and acidified water supplemented with Bactrim (a combination of trimethoprim and sulfametoxazole suspension, $60 \mathrm{mg} / \mathrm{kg} / \mathrm{day}$, dose based on the concentration of sulfa) were given to the bone marrow cells recipient $N k x 2-1(f l / f)$;TPO-cre mice starting 1 week prior to irradiation, and continued for two weeks after irradiation. Thyroids of these mice were collected at 8 weeks post-bone marrow transplantation.

\section{Histological analyses}

Thyroids were fixed in $10 \%$ buffered formalin, embedded in paraffin, and sectioned at $4 \mu \mathrm{m}$. Sections were deparaffinized and stained with hematoxylin and eosin (H\&E). For immunohistochemistry and immunofluorescence, sections were treated with 5\% hydrogen peroxide in PBS for 5 min to block endogenous peroxidase activity, followed by rinsing for $5 \mathrm{~min}$ with PBS. Epitope retrieval was carried out by autoclaving for $15 \mathrm{~min}$ in citrate buffer, $\mathrm{pH}$ 7.0. After cooling to room temperature, the sections were incubated overnight at $4{ }^{\circ} \mathrm{C}$ with the following primary antibodies; anti-NKX2-1 (rabbit polyclonal, 1:1000, H-190, sc-13040, Santa Cruz Biotechnology), anti-PAX8 (rabbit polyclonal, 1:1000, 10336-1-AP, Proteintech), anti-GFP (chicken polyclonal 1:1000 ab13970, Abcam), anti-E-cadherin (rabbit monoclonal, 1:500, 24E10, \#3195. Cell Signaling Technology), anti-Thyroglobulin (goat polyclonal, 1:100, sc-7836, Santa Cruz Biotechnology), anti-BrdU (rat polyclonal, 1:100, OBT0030, Serotec), and anti-pAKT (Ser473) (rabbit monoclonal, 1:100, 193H12, \#4058, Cell Signaling Technology). For immunohistochemistry, the sections were washed with PBS, followed by staining using the ABC method with a commercially available kit (Vector Laboratories) according to the manufacturer's instructions. Immunostaining was visualized with 3,3'-diaminobenzidine as substrate (Sigma-Aldrich), and counterstained with hematoxylin. For immunofluorescence, sections were treated with the following secondary antibodies; labeled goat anti-rabbit IgG antibodies (Alexa Fluor 594, 1:800, Invitrogen, and Alexa Fluor 488, 1:400, Invitrogen), labeled goat anti-chicken IgG antibodies (Alexa Fluor 488, 1:400, Invitrogen), labeled donkey anti-goat IgG (Alexa Fluor 488, 1:400, Invitrogen), and labeled donkey anti-rabbit $\operatorname{IgG}$ (Alexa Fluor 594, 1:800, Invitrogen) for $1 \mathrm{~h}$ at room temperature and washed with PBS. For double staining using two primary antibodies from the same host species, 
unconjugated Fab fragments were used for blocking after the first secondary antibody. For example, multi-staining (NKX21, PAX8) was performed as follows. After epitope retrieval and blocking of the non-specific binding sites, sections were incubated with the first primary antibody (NKX2-1) for $1 \mathrm{~h}$ at room temperature. After washing with $\mathrm{PBS}$, the sections were incubated with the first secondary antibody (Alexa Fluor 594 goat anti-rabbit $\operatorname{IgG}$ ) and washed with PBS. Sections were then incubated with normal serum (5\% rabbit serum) from the same host species as the first primary antibodies for $1 \mathrm{~h}$ at room temperature and washed with PBS. Sections were further incubated with an excess of unconjugated Fab antibody (AffiniPure Fab fragment goat anti-rabbit IgG, Jackson Immuno Research Laboratory) derived from the same host species as the primary antibody for $1 \mathrm{~h}$ at room temperature and washed with PBS. The sections were finally incubated with the second primary antibody (PAX8) overnight at $4{ }^{\circ} \mathrm{C}$, washed with PBS, and were incubated with the second secondary antibody (Alexa Fluor 488 goat anti-rabbit IgG) for 1 $\mathrm{h}$ at room temperature and washed with PBS. For multistaining using Fab, primary and secondary antibodies were used at different dilutions from those used for single staining. DAPI dye (Life Technologies-Thermo Fisher Scientific) was used to stain the cell nuclei. Confocal images were obtained with a BZ-X700 (KEYENCE).

\section{Western blotting}

SPTL and mouse thyroid follicular cells were lysed with RIPA Lysis Buffer (MilliporeSigma) with proteinase inhibitor cocktail, cOmplete mini EDTA-Free (Roche). The Pierce BCA Protein Assay Kit (Thermo Fisher Scientific) was used for measuring the protein concentration of lysates. Samples were subjected to SDS-PAGE and transferred to PVDF membranes (GE Healthcare). Tris-buffered saline buffer containing 5\% bovine albumin fraction V (MP Biomedicals) and $0.1 \%$ Tween-20 (Sigma-Aldrich) was used for blocking. Stripping of membrane was performed using Restore Western Blot Stripping Buffer (Thermo Fisher Scientific). Antibodies used were those against AKT (\#9272, Cell Signaling Technology), phospho-p44/p42 MAPK (ERK1/2) (Thr202/ Thy204) (\#9101, Cell Signaling Technology), ERK1+ERK2 (ab17942, Abcam), PAX8 (clone PAX8R1, ab53490, Abcam), $\alpha$-tubulin (clone DM1A, sc-32293, Santa Cruz), and $\beta$-actin (clone D6A8, \#8457, Cell Signaling Technology). Anti-NKX2-1, anti-E-cadherin, and anti-phospho-AKT (Ser473) antibodies were the same as those used for immunohistochemistry/immunofluorescence.

\section{Construction of SPTL cells overexpressing NKX2-1}

The details of mouse thyroid side population-derived cell line, SPTL cells were previously described [17]. Rat Nkx2-1 was subcloned into pENTR/D-TOPO (Life Technologies) and transferred by Gateway LR Clonase II enzyme mix (Life Technologies) to pInducer20 (Addgene, \#44012). Rat NKX2-1 differs only 2 amino acid residues from mouse protein in its sequence [23]. Lentivirus was generated using $293 \mathrm{~T}$ cells (ATCC), psPAX2 (Addgene, \#12260), and pMD2.G (Addgene, \#12259). Selection was initiated at $48 \mathrm{~h}$ after infection by the addition of $200 \mu \mathrm{g} / \mathrm{ml}$ of $\mathrm{G} 418$ (Thermo Fisher Scientific).

\section{Results}

\section{Characterization of Nkx2-1(fl/fl);TPO-cre mouse thyroid}

As compared with normal mouse thyroid, which consists of many round small follicles of relatively uniform shape (Fig. 1a), Nkx2-1(fl/fl);TPO-cre mouse thyroids had a very disorganized appearance, consisting of vastly different sized follicles including extremely large follicles of irregular shape (Fig. 1b). Most retained colloid inside their follicles. Some follicular epithelia were focally stratified while other follicular cells were very thin and almost degenerated (Fig. 1b-d). In these thin follicular cells, PAX8 and thyroglobulin expression was found, but no NKX2-1 expression observed (Fig. 1e). This is in good agreement with the original description of $N k x 2-1(f / / f)$;TPO-cre mouse thyroids [11]; follicular cells that have lost NKX2-1 expression appear to undergo degeneration.

\section{Presence of NKX2-1-positive cells in non-follicular areas}

There were many small irregular shape follicles observed close to the tracheal cartilage and muscle (Fig. 1d). Some of the small irregular shape follicles looked as if they were under folliculogenesis (Fig. 1d, f). Some were clustered in nest, while others were about to divide into two or three follicles, or as if a small follicle was about to bud out from the parent follicle (Fig. 1f). Upon careful examination of NKX2-1 expression patterns by immunohistochemistry, a thin zone of NKX2-1 positive cells were noted lining near the tracheal cartilage and muscle (Fig. 1d, f, g). This zone of cells, many of which had NKX2-1 expression, were thinner and elongated in shape as compared with the round shape NKX2-1-expressing follicular cells (Figs. 1g, 2a). A newly formed follicle-like structure was found connected at the end or in the middle of the zone of elongated cells (Fig. 2a, b). Cells of the newly formed follicle-like structures expressed PAX8, the epithelial marker E-cadherin, and one of the thyroid differentiation markers thyroglobulin, indicating that they are indeed thyroid follicles. 


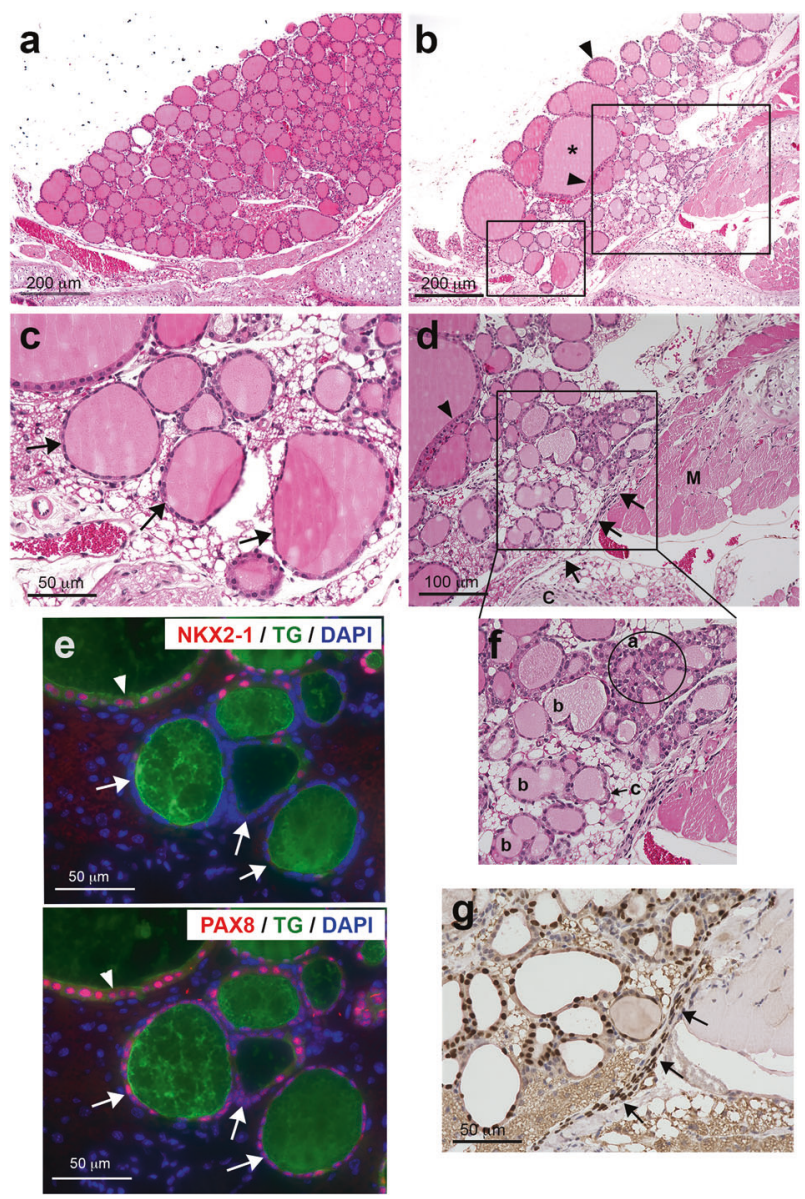

Fig. $1 N k x 2-1(f / / f)$; TPO-cre mouse thyroid. a-d, f H\&E staining of control (a) and Nkx2-1(fl/fl);TPO-cre mouse thyroid (b). Asterisk: a large follicle in irregular shape. Arrowheads: focally stratified follicular epithelial cells. $\mathbf{c}$ High magnification of a small box shown in $\mathbf{b}$. Arrows: follicular cells undergoing degeneration. d High magnification of a large box shown in b. Arrows: a zone of follicular precursor cells that are NKX2-1-positive (g). Arrowhead: focally stratified follicular epithelial cells. C: cartridge, M: muscle. e Immunofluorescence using nearby sections to $\mathbf{c}$ for NKX2-1 and thyroglobulin (TG) (upper), and PAX8 and TG (lower). Arrowhead: normal follicular cells express both NKX2-1 and PAX. Arrows: follicular cells undergoing degeneration that are thin and do not express NKX2-1. f High magnification of a box shown in d. Many types of folliculogenesis are found; a: solid nest type, b: dividing type, c: budding type. g Immunohistochemistry for NKX2-1 of the area shown in f. Arrows: a zone of NKX2-1-positive cells

NKX2-1-positive cells were widespread within the zone of elongated cells, while strongly PAX8-positive cells were located only near the appeared-to-be newly formed follicles (Fig. 2b, c). This suggests the possibility that NKX2-1 expression is critical and is a prerequisite for PAX8 expression; both NKX2-1 and PAX8 are required for functional follicle formation and thyroglobulin expression [20-22]. Compared with many NKX2-1-positive cells, only a few cells were BrdU positive, suggesting that the zone of elongated cells are not growing, but rather in a fully differentiation mode (Fig. 2d). When Nkx2-1(fl/fl);TPO-cre mice were subjected to a bone marrow transplant experiment using bone marrow cells obtained from GFPtransgenic mice, some GFP-positive cells were found surrounding NKX-2-1-positive cells within the zone of cells (Fig. 2a, Supplementary Figure 1). These results suggest that a zone of clustered cells could be, at least partly, derived from bone marrow cells.

\section{Induction of phosphorylation of AKT by NKX2-1}

SPTL cells are considered to be immature, partially thyroid lineage-committed progenitor cells, of which approximately $1.3 \%$ of cells express NKX2-1, but not other thyroid differentiation markers including PAX8 when cultured under low-serum differentiation conditions [17]. NKX2-1 is critical for thyroid lineage specification [18, 19], however, both NKX2-1 and PAX8 are necessary for thyroid committed cells to become follicular cells [20-22]. In order to determine whether high levels of NKX2-1 expression drives PAX8 expression in SPTL cells, doxycycline-inducible NKX2-1-overexpressing SPTL cells were established (Fig. 3a). The expression of NKX2-1 was detected by the simultaneous expression of GFP. NKX2-1 was robustly expressed in most of cells when they were treated with doxycycline at $1 \mu \mathrm{g} / \mathrm{ml}$, while no PAX8 expression was found (Fig. 3a, b), suggesting that NKX2-1 expression is not sufficient to drive PAX8 expression in SPTL cells at least under the culture conditions used. The absence of follicular cell differentiation was evident from the lack of Ecadherin expression in NKX2-1-overexpressing SPTL cells. These NKX2-1-overexpressing cells under doxycycline exhibited a different morphology having more elongated shape as compared with non-NKX2-1-expressing cells, and without an apparent increase in growth rate (Fig. 3c). In order to determine the signaling events occurring in NKX21-overexpressing SPTL cells, several signaling molecules were examined by western blotting (Fig. 3d). Interestingly, phosphorylation of AKT was induced as NKX2-1 expression increased, while phosphorylation of ERK remained unchanged or rather decreased. These results suggest that AKT phosphorylation may be downstream of NKX2-1 expression, but preceding PAX8 expression during thyroid follicular cells differentiation. Indeed, a few pAKT-positive cells were found co-expressing NKX2-1 within the cluster of NKX2-1-positive non-follicular precursor cells near the tracheal cartilage and muscle (Fig. 3e).

\section{Discussion}

In this study, the $N k x 2-1(f / f l)$;TPO-cre mouse was employed as a unique model for studying in vivo thyroid 

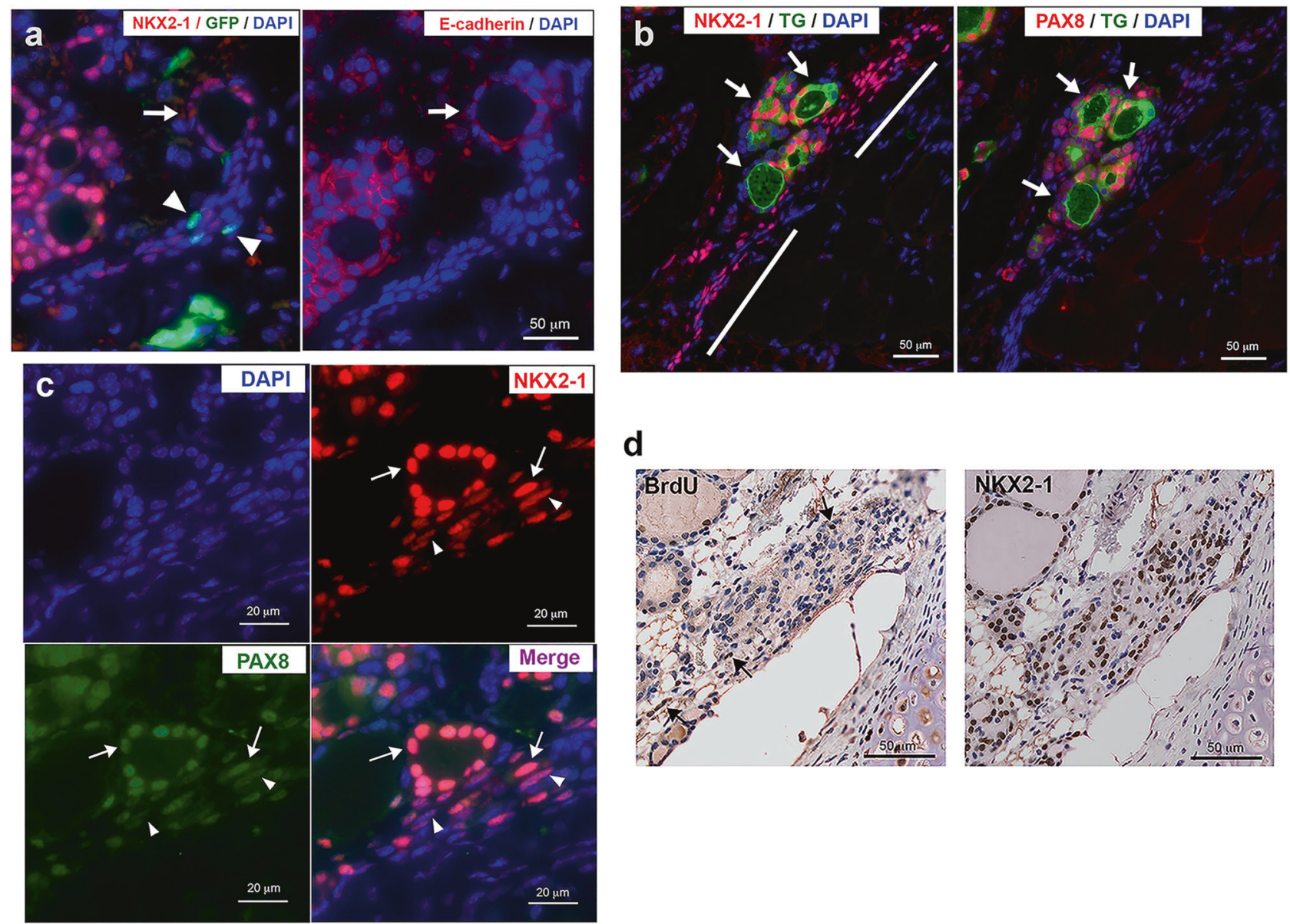

d

Fig. 2 Formation of thyroid follicles from NKX2-1-positive nonfollicular cells. a Immunofluorescence for NKX2-1 and GFP (left), and E-cadherin (right) in the $N k x 2-1(f / / f)$; TPO-cre mice that received bone marrow cells from GFP-transgenic mice. Arrows: a newly formed follicle connected to a zone of cells containing many NKX2-1-positive cells. Arrowheads: GFP-positive cells. b Immunofluorescence for NKX2-1 and TG (left), and PAX8 and TG (right). Arrows: newly

formed follicles located in the middle of a zone of cells containing many NKX2-1-positive cells, but very weak PAX8 expression (shown by bars). c Immunofluorescence for NKX2-1 and PAX8 near a newly formed follicle. Arrows: NKX2-1 and PAX8 both strongly expressed. Arrowheads: PAX8 expression is weaker than NKX2-1, resulting in stronger magenta color in the merged image. d Immunohistochemistry for BrdU (left) and NKX2-1 (right). Arrows: BrdU positive cells

regeneration and folliculogenesis. An area consisting of NKX2-1-positive immature follicular cell precursors was found close to the tracheal cartilage and muscle. These cells appear to be the source of newly produced follicles in the thyroid. Others described three types of thyroid follculogenesis in vitro using thyroid cells cultured on threedimensional collagen gel; solid nest, lumen-dividing, and budding type [24]. These three types of folliculogenesis were indeed observed in the $N k x 2-1(f / f l)$;TPO-cre mouse thyroid. A cluster of cells containing NKX2-1-positive thyroid follicular precursor cells are rarely found in normal mouse thyroids because they are in much smaller areas and are not as conspicuous as those in $N k x 2-1(f / f l)$;TPO-cre mice. This is probably because follicular cells in the $N k \times 2-1$ ( $f(f)$ );TPO-cre mouse thyroid have high turnover rates and constantly undergo repeated degeneration and regeneration; cells that have lost NKX2-1 degenerate while follicular cells

constantly regenerate in order to maintain thyroid hormone levels $[11,12]$. Whether a group of thyroid follicular precursor cells have the limited numbers that are initially developmentally set during embryonic thyroid development and are exhausted after repeated regeneration, or can be replenished as needed remains to be determined. While newly formed follicles that express GFP were not observed, the present results suggest the possibility that these precursor cells might be replenished, at least partly, by bone marrow derived cells.

We previously proposed a two-step process of thyroid folliculogenesis; thyroid follicle formation requires NKX21 expression, while PAX8 is required for the formation of functional thyroid follicles [17]. The current results suggest that pAKT expression may be the next step to NKX2-1 expression, but before PAX8 expression during thyroid follicular cell differentiation. The reason why PAX8 is not 
Fig. 3 Signaling in NKX2-1overexpressing cells. a GFP immunofluorescence to determine the efficiency of NKX2-1 overexpression in the presence or absence of doxycycline (DOX, $1 \mu \mathrm{g} / \mathrm{ml}$ ) in SPTL cells. b Western blotting for thyroid and epithelial differentiation markers in SPTL cells cultured for 2 days under $0.1 \%$ FBS without $(-)$ and with (+) DOX $(1 \mu \mathrm{g} / \mathrm{ml})$. Thy: mouse thyroid follicular cells. $\beta$-actin was used as a loading control. c Cell morphology of SPTL cells cultured under DOX. Images were taken after 3 days culture under $2 \%$ FBS in the absence or presence of DOX $(3 \mu \mathrm{g} / \mathrm{ml})$. d Western blotting for DOX dosedependent expression of NKX21, ERK, pERK, AKT, and pAKT in SPTL cells. Cells were cultured under $0.1 \%$ FBS and treated with DOX for 2 days. $10 \%$ FBS for 1.3 hours was used as positive control for pAKT. $\alpha$ tubulin was used as a loading control. e Immunofluorescence for NKX2-1 and pAKT in a zone of cells located near the tracheal cartilage and muscle in Nkx2-1(fl/fl);TPO-cre mouse. Arrows: cells expressing both pAKT and NKX2-1 a
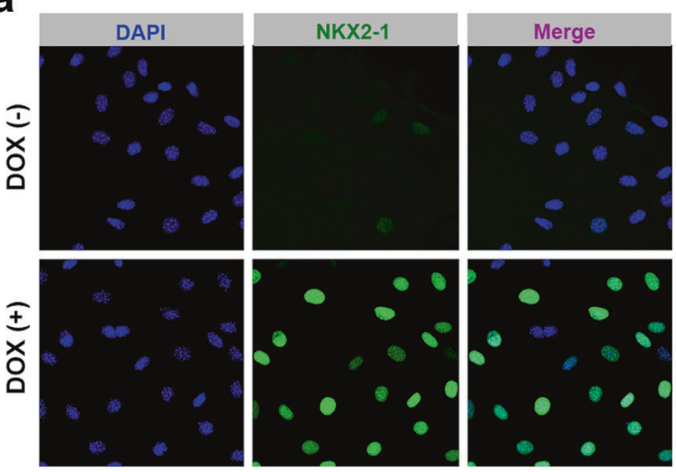

C
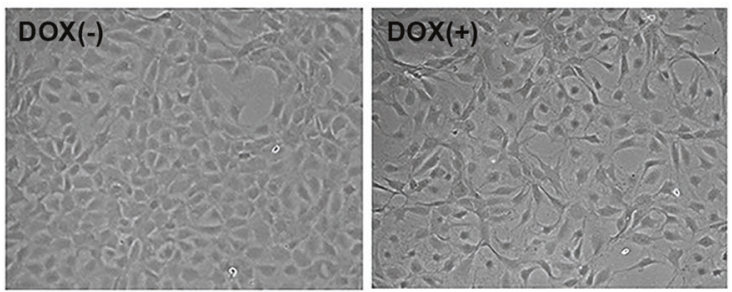

b SPTL-NKX2-1

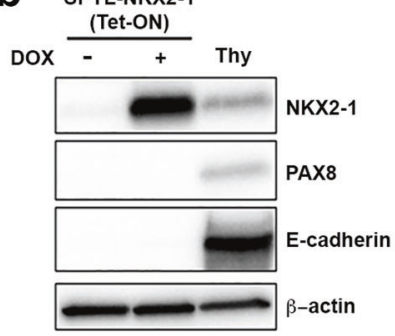

d SPTL-NKX2-1 (Tet-ON)
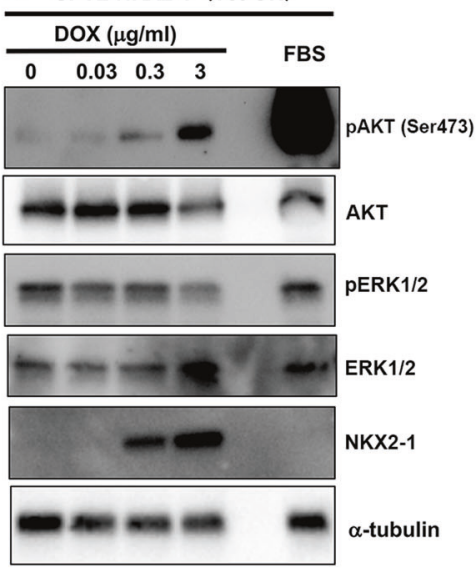

e DAPI

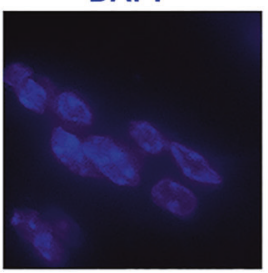

NKX2-1

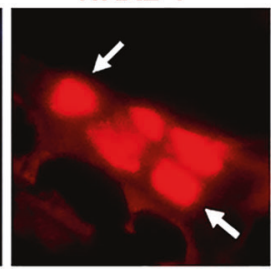

pAKT
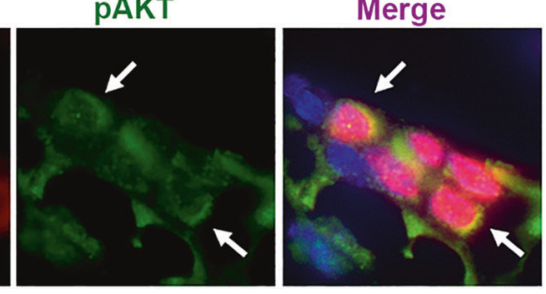

expressed in NKX2-1-overexpressing SPTL cells is currently not known. Possibly signal(s) other than pAKT may be required for PAX8 expression, at least under the culture conditions used in vitro. Alternatively, SPTL cells may be intrinsically missing the capacity to proceed to further differentiation. Related to this, it was previously reported using lung adenocarcinoma cells that phosphorylation of AKT is induced by NKX2-1 through receptor tyrosine kinase-like orphan receptor 1 (ROR1) [25]. Additional studies are required to address these questions.

It is well known that phosphatidylinositide 3-kinase (PI3K)/AKT signaling pathway plays a key role in multiple cellular processes, such as energy metabolism, apoptosis, proliferation, and cell migration [26]. In the thyroid, insulin/ IGF-1 through PI3K/AKT signaling regulates thyroid cell growth and cell cycle progression [27]. In the current study, it is likely that NKX2-1-positive cells are not in the active proliferation state according to the results of BrdU staining, even if some cells express pAKT. Further, NKX2-1overexpressing SPTL cells showed no apparent increased proliferation even though AKT phosphorylation was enhanced. These results suggest that NKX2-1 may directly regulate some genes essential for cell growth arrest, which is dominant over the growth promoting AKT phosphorylation signaling. Moreover, AKT phosphorylation was reported to be critical for the differentiation of muscle [28], osteoblast, and bone development [29]. Thus, it is reasonable to assume that pAKT signaling may play some role in the early stages of thyroid differentiation. However, the involvement of pAKT in thyroid differentiation has not been documented. Whether this is the case, and if so, the mechanism of action awaits further studies.

Recent studies revealed that thyroids exhibit compensatory enlargement after hemithyroidectomy in humans, in which the remaining thyroid lobe showed the increased proliferation and Ki67 immunostaining [30, 31]. Whether precursor cells to thyroid follicular cells are also present in humans as described herein in mice, and if so where they are located, are not known. However, the current study may suggest that it would be preferable to 
leave some unresected tissues, particularly those aligning near the tracheal cartilage and muscle when thyroidectomy is performed. There remains the possibility that the residual thyroid tissue may undergo active regeneration.

In conclusion, $N k x 2-1(f / f)$ ); TPO-cre mouse thyroid provides a suitable in vivo model to study thyroid regeneration and folliculogenesis. A zone of NKX2-1-positive cells located near the tracheal cartridge and muscle may be follicular cell precursors, from which mature follicles are formed.

Acknowledgements This work was supported by the National Cancer Institute Intramural Research Program, Center for Cancer Research ZIABC005522 (to SK), and JSPS (Japan Society for the Promotion of Science) Research Fellowship for Japanese Biomedical and Behavioral Researchers at NIH (to YT).

\section{Compliance with ethical standards}

Conflict of interest The authors declare that they have no conflict of interest.

\section{References}

1. Coclet J, Foureau F, Ketelbant P, et al. Cell population kinetics in $\operatorname{dog}$ and human adult thyroid. Clin Endocrinol. 1989;31: 655-65.

2. Capen CC. Mechanistic data and risk assessment of selected toxic end points of the thyroid gland. Toxicol Pathol. 1997;25: 39-48.

3. Capen CCMartin SL. The effects of xenobiotics on the structure and function of thyroid follicular and C-cells. Toxicol Pathol. 1989;17:266-93.

4. Chen CY, Kimura H, Landek-Salgado MA, et al. Regenerative potentials of the murine thyroid in experimental autoimmune thyroiditis: role of CD24. Endocrinology. 2009;150:492-9.

5. Johansen R, Gardner RE, Galante M, et al. An experimental study of thyroid regeneration following subtotal thyroidectomy. Surg Gynecol Obstet. 1951;93:303-9.

6. Okamoto M, Hayase S, Miyakoshi M, et al. Stem cell antigen 1positive mesenchymal cells are the origin of follicular cells during thyroid regeneration. PLoS One. 2013;8:e80801.

7. Ozaki T, Matsubara T, Seo D, et al. Thyroid regeneration: characterization of clear cells after partial thyroidectomy. Endocrinology. 2012;153:2514-25.

8. Kimura S. Thyroid regeneration: how stem cells play a role? Front Endocrinol. 2014;5:55

9. Lee J, Yi S, Chang JY, et al. Regeneration of thyroid follicles from primordial cells in a murine thyroidectomized model. Lab Invest. 2017;97:478-89.

10. Kusakabe T, Kawaguchi A, Kawaguchi R, et al. Thyrocytespecific expression of Cre recombinase in transgenic mice. Genesis. 2004;39:212-6.

11. Kusakabe T, Kawaguchi A, Hoshi N, et al. Thyroid-specific enhancer-binding protein/NKX2.1 is required for the maintenance of ordered architecture and function of the differentiated thyroid. Mol Endocrinol. 2006;20:1796-809.
12. Hoshi S, Hoshi N, Okamoto M, et al. Role of NKX2-1 in N-bis(2hydroxypropyl)-nitrosamine-induced thyroid adenoma in mice. Carcinogenesis. 2009;30:1614-9.

13. Hoshi N, Kusakabe T, Taylor BJ, et al. Side population cells in the mouse thyroid exhibit stem/progenitor cell-like characteristics. Endocrinology. 2007;148:4251-8.

14. Thomas T, Nowka K, Lan L, et al. Expression of endoderm stem cell markers: evidence for the presence of adult stem cells in human thyroid glands. Thyroid. 2006;16:537-44.

15. Lan L, Cui D, Nowka K, et al. Stem cells derived from goiters in adults form spheres in response to intense growth stimulation and require thyrotropin for differentiation into thyrocytes. J Clin Endocrinol Metab. 2007;92:3681-8.

16. Fierabracci A, Puglisi MA, Giuliani L, et al. Identification of an adult stem/progenitor cell-like population in the human thyroid. J Endocrinol. 2008;198:471-87.

17. Murata T, Iwadate M, Takizawa $Y$, et al. An adult mouse thyroid side population cell line that exhibits enriched epithelialmesenchymal transition. Thyroid. 2017;27:460-74.

18. Dame K, Cincotta S, Lang AH, et al. Thyroid progenitors are robustly derived from embryonic stem cells through transient, developmental stage-specific overexpression of Nkx2-1. Stem Cell Rep. 2017;8:216-25.

19. Kurmann AA, Serra M, Hawkins F, et al. Regeneration of thyroid function by transplantation of differentiated pluripotent stem cells. Cell Stem Cell. 2015;17:527-42.

20. Antonica F, Kasprzyk DF, Opitz R, et al. Generation of functional thyroid from embryonic stem cells. Nature. 2012;491:66-71.

21. Ma R, Latif RDavies TF. Human embryonic stem cells form functional thyroid follicles. Thyroid. 2015;25:455-61.

22. Ma R, Morshed SA, Latif R, et al. Thyroid cell differentiation from murine induced pluripotent stem cells. Front Endocrinol. 2015;6:56.

23. Oguchi H, Pan YTKimura S. The complete nucleotide sequence of the mouse thyroid-specific enhancer-binding protein (T/EBP) gene: extensive identity of the deduced amino acid sequence with the human protein. Biochim Biophys Acta. 1995;1261:304-6.

24. Toda S, Aoki S, Suzuki K, et al. Thyrocytes, but not C cells, actively undergo growth and folliculogenesis at the periphery of thyroid tissue fragments in three-dimensional collagen gel culture. Cell Tissue Res. 2003;312:281-9.

25. Yamaguchi T, Yanagisawa K, Sugiyama R, et al. NKX2-1/TITF1/ TTF-1-induced ROR1 is required to sustain EGFR survival signaling in lung adenocarcinoma. Cancer Cell. 2012;21:348-61.

26. Vanhaesebroeck B, Stephens LHawkins P. PI3K signalling: the path to discovery and understanding. Nat Rev Mol Cell Biol. 2012;13:195-203.

27. Saito J, Kohn AD, Roth RA, et al. Regulation of FRTL-5 thyroid cell growth by phosphatidylinositol $(\mathrm{OH}) 3$ kinase-dependent Aktmediated signaling. Thyroid. 2001;11:339-51.

28. Rotwein PWilson EM. Distinct actions of Akt1 and Akt2 in skeletal muscle differentiation. J Cell Physiol. 2009;219:503-11.

29. Mukherjee ARotwein P. Akt promotes BMP2-mediated osteoblast differentiation and bone development. J Cell Sci. 2009;122:716-26.

30. Bychkov A. Epithelial hyperplasia is responsible for the compensatory enlargement of remaining thyroid lobe after thyroidectomy. Eur Arch Otorhinolaryngol. 2018. https://doi.org/10. 1007/s00405-018-4915-6.

31. Pustelnik FS, Gronbek C, Dossing H, et al. The compensatory enlargement of the remaining thyroid lobe following hemithyroidectomy is small and without impact on symptom relief. Eur Arch Otorhinolaryngol. 2018;275:161-7. 